\title{
Function of Aurora kinase A in Taxol-resistant breast cancer and its correlation with P-gp
}

\author{
YAN LI, KE TANG, HAIJING ZHANG, YI ZHANG, WANQI ZHOU and XIAOGUANG CHEN \\ Department of Pharmacology, Institute of Materia Medica, Chinese Academy of Medical Sciences \\ and Peking Union Medical College, Beijing 100050, P.R. China
}

Received February 28, 2011; Accepted May 12, 2011

DOI: $10.3892 / \mathrm{mmr} .2011 .494$

\begin{abstract}
Breast cancer is one of the most common malignant diseases among women. In early and metastatic breast cancer, Taxane (Taxol) is widely used as an adjuvant and neoadjuvant therapies. Although breast cancer is initially responsive to Taxol, inherent or developed resistance to Taxol often limits the efficacy of the drug. The oncogene Aurora kinase A is frequently up-regulated in human cancer, and is associated with sensitivity to chemotherapy in certain types of cancer. In the present study, we aimed to clarify the functional role of Aurora kinase A in breast cancer resistance to Taxol, and to determine the means to overcome this resistance. The correlation between the expression levels of Aurora kinase A and chemoresistance to Taxol in breast cancer cells, and resistance to Taxol in a xenograft model were demonstrated. MTT assay was performed to determine cell viability. Subsequently, the relationship of Aurora kinase A with the expression and functional role of P-gp was clarified, as well as its relationship with $\mathrm{p}$-ERK2, which regulates the expression of P-gp. The expression of Aurora A was determined to be capable of enhancing the sensitivity of cells resistant to Taxol in vitro and in vivo using stable knockdown Aurora kinase A cells. We propose that this kinase may be used as a target for overcoming chemoresistance and enhancing the chemosensitivity of breast cancer to Taxol.
\end{abstract}

\section{Introduction}

Breast cancer is one of the most common malignant diseases among Western women (1). Taxane (Taxol) and docetaxel (Taxotere) are widely used in early and metastatic breast cancer as adjuvant and neoadjuvant therapies (2). Although initially responsive in breast cancer, inherent or developed Taxol resistance often limits the efficacy of Taxol $(3,4)$. Overexpression

Correspondence to: Dr Xiaoguang Chen, Department of Pharmacology, Institute of Materia Medica, Chinese Academy of Medical Sciences and Peking Union Medical College, 1 Xian Nong Tan Street, Beijing 100050, P.R. China

E-mail: chxg@imm.ac.cn

Key words: Taxol, Aurora A, chemoresistance, breast cancer, P-gp and activity of drug efflux pumps, such as P-gp, and changes in the $\beta$-tubulin isoform mainly confer the resistance, which can be identified in the clinic. Thus, prevention from P-gp overexpression and inhibition of the action may improve the effectiveness of chemotherapy. However, the molecular mechanisms regarding the regulation of $\mathrm{P}$-gp expression have yet to be adequately clarified (4).

Aurora kinases are a family of serine-threonine kinases that act as conserved mitotic regulators. Aurora B and C are expressed in mammalian cells. Aurora A and B are the best characterized, and regulate distinct processes in mitosis. During mitosis, Aurora A localizes to the centrosomes and spindle poles. It is also involved in the centrosome maturation and separation and in the assembly of the mitotic spindle. By contrast, Aurora B localizes to the centromeres during the early stages of mitosis, which is thought to catalyze the attachment of chromosomes to microtubules, the spindle checkpoint and cytokinesis. Expression of Aurora $\mathrm{C}$ is restricted to germ cells, in which it is believed to regulate spermatogenesis. Aurora kinase A is an oncogene frequently elevated in human cancers and is associated with advanced clinical staging and poor survival (5). Currently, a variety of ATP-competitive inhibitors that target Aurora kinases and possess varying degrees of kinase specificity are utilized in early clinical development (6).

An association between the high expression of Aurora kinases and chemosensitivity to docetaxel has been reported in prostate cancer cell lines in vitro (7). This suggests that high expression of Aurora A is also associated with resistance to Taxol in breast cancers. However, whether Aurora kinase A is involved in chemoresistance to Taxol in breast cancer has yet to be investigated, particularly its correlation to P-gp. Considering these findings, clarifying this correlation of Aurora A to enhance sensitivity to Taxol resistance in breast cancer would be of great interest. Therefore, in the present study, the correlation between expression levels of Aurora kinase A and chemoresistance to Taxol in breast cancer cells, and xenograft resistance to the Taxol model was investigated. Additionally, the study aimed to clarify the relationship of Aurora kinase A with P-gp and with p-ERK2, which is reported to regulate the expression of P-gp. Decreasing the expression of Aurora A was determined as able to enhance the sensitivity of resistant cells to Taxol in vitro and in vivo using stable knockdown Aurora kinase A cells. The possible 
application of this kinase as a means of overcoming chemoresistance and enhancing chemosensitivity of breast cancer to Taxol is proposed.

\section{Materials and methods}

Cell culture. An MCF-7 cell line was obtained from the cell center of CAMS and PUMC. MX-1 and MX-1/Taxol cells were kindly provided by Professor Yongkui Jing (Mount Sinai School of Medicine, New York, NY, USA). The cells were maintained in DMEM or RPMI-1640 (Invitrogen, Carlsbad, CA, USA) supplemented with $10 \%$ fetal bovine serum (FBS; Gibco, Carlsbad, CA, USA), $100 \mathrm{IU} / \mathrm{ml}$ penicillin and $100 \mu \mathrm{g} /$ $\mathrm{ml}$ streptomycin in a humidified incubator containing $5 \% \mathrm{CO}_{2}$ at $37^{\circ} \mathrm{C}$.

Establishment of Taxol-resistant cell line. MCF-7/Taxol was produced by repeatedly exposing MCF-7 cells to Taxol (synthesized by Professor Weishuo Fang in CAMS and PUMC) at a single high concentration. In brief, MCF-7/Taxol was selected for a procedure consisting of 18 pulse drug treatments with Taxol. The majority of the cells were dead following $24 \mathrm{~h}$ of exposure to Taxol. The treated cells were then washed with $0.01 \mathrm{~mol} / 1$ phosphate-buffered saline (PBS) and cultured in Taxol growth medium. After 1-2 days, the dead cells were washed out with PBS and fresh Taxol medium was added. Once the cells reached $70-80 \%$ confluence, they were preserved for further study. The Taxol-resistant cell line was stabilized for approximately 6 months after treatment initiation, and the resistant phenotype was developed. For the maintenance of Taxol-resistant cells, the MCF-7/Taxol cells were grown in the presence of $0.001 \mu \mathrm{mol} / 1 \mathrm{Taxol}$. Prior to experimentation, MCF-7/Taxol cells were maintained in a Taxol-free culture medium and subcultured for at least three generations. The drug-resistant characteristics of the MCF-7/ Taxol cells were tested using various concentrations of Taxol.

Cell viability assay. A total of MCF-7, MCF-7/Taxol, MX-1, MX-1/Taxol and other sublines were seeded in a 96-well plate. After $24 \mathrm{~h}$, the cells were treated with different concentrations of Taxol. After $96 \mathrm{~h}$ of incubation, the MTT (Sigma, St. Louis, MO, USA) assay was performed to evaluate cell viability. Absorbance values were normalized to the values obtained for vehicle-treated cells to determine the percentage of surviving cells. Each assay was performed in triplicate.

Accumulation of Rhol 23 was measured by flow cytometry. The measurement of Rho123 (Sigma) accumulation was performed by the flow cytometric assay (FCM). In brief, cells $\left(5 \times 10^{5}\right.$ per sample) were incubated with $10 \mu \mathrm{mol} / 1$ of Rhol23 in the dark at $37^{\circ} \mathrm{C}$ in $5 \% \mathrm{CO}_{2}$ for $180 \mathrm{~min}$. Verapamil $(10 \mu \mathrm{mol} / \mathrm{l})$ was then added to the cultures at the same time as Rho123. Following Rho123 accumulation, cells were washed twice with ice-cold PBS and placed in PBS with 10\% FBS on ice. The green fluorescence of Rho123 was measured by flow cytometry, and the fluorescence values were measured using excitation and emission wavelengths of 480 and $540 \mathrm{~nm}$, respectively.

Western blot analysis. Lysates were prepared by washing cells with PBS according to the methods previously described (8).
For Western blotting, samples transferred to a nitrocellulose membrane by semi-wet electrophoresis were subjected to SDS-PAGE and incubated with primary antibody [rabbit anti-Aurora A, rabbit anti-phosphorylated Aurora A (Thr288), mouse anti-phosphorylated Histone H3 (Ser10), rabbit antiERK2 and rabbit anti-phosphorylated ERK2)] (Santa Cruz Biotechnology, Santa Cruz, CA, USA) antibodies overnight at $4^{\circ} \mathrm{C}$. The samples were detected with horseradish peroxidase-conjugated anti-rabbit or anti-mouse IgG (Santa Cruz Biotechnology), and developed using an ECL Western blot detection and analysis system (Applygen Technologies Inc., Beijing, China). Membranes were tested for equal loading by probing for actin.

Establishment of the Taxol-resistant MX-1 xenograft model. An in vivo MX-1 xenograft model (MX-1/Taxol) resistant to Taxol was established in order to study resistance mechanisms acquired in a therapeutic setting. Athymic nude mice (Balb/c-nu/nu females, 6-8 weeks old) were purchased from Vital River Laboratory Animal Technology Co., Ltd. (Beijing, China) and housed in controlled environment at $25^{\circ} \mathrm{C}$ on a 12-h light/dark cycle. Mice were maintained in accordance with the National Institute of Health Guide for the Care and Use of Laboratory Animals. Tumor resistance was achieved in the flanks of Athymic nude mice after eight passages (treated with $30 \mathrm{mg} / \mathrm{kg} /$ day x 1 Taxol every 14-day passage). Resistance was characterized by the absence of tumor regression and a significant decrease of tumor-doubling time.

Tissue culture of MX-1 from Taxol-resistant MX-1 xenograft model. Tumor tissues from a Taxol-resistant MX-1 xenograft model for eight generations and a parent MX-1 xenograft model were cut into small pieces with surgical scissors and minced with sterile razor blades until the explant size was $<1 \mathrm{~mm}^{3}$. The explants were transferred to flasks, trypsinized (Invitrogen) for $30 \mathrm{~min}$, covered with complete medium and incubated in an atmosphere of $5 \% \mathrm{CO}_{2}$ and $95 \%$ air at $37^{\circ} \mathrm{C}$. The medium was replaced after $48 \mathrm{~h}$.

Generation of the stable knockdown Aurora A in the MX-1/ Taxol cell line. MX-1/Taxol cells were seeded at a density of $1 \times 10^{5}$ cells per well in 6-well plates. Following overnight incubation, the cells reached $50 \%$ confluence. The cells were then transfected with either the Aurora kinase A miRNA or control miRNA vector (BLOCK-iT ${ }^{\mathrm{TM}}$ Pol II miR RNAi Expression Vector kit with EmGFP, purchased from Invitrogen) using Lipofectamine 2000 (Invitrogen), according to the manufacturer's recommended protocol. In brief, the normal cellular medium was replaced with serum- and antibody-free DMEM medium. The miRNA vectors and Lipofectamine mixture were added directly to the six plates without removal of the culture medium. The medium was mixed by gentle agitation. The plates were incubated for an additional $6 \mathrm{~h}$ and then changed to normal medium. The initial selection for transfected cells was performed by growth in fresh medium containing $8 \mu \mathrm{g} /$ $\mathrm{ml}$ Blasticidin $\mathrm{S} \mathrm{HCl}$ (Invitrogen). Selective pressure was maintained by growth in a medium containing $8 \mu \mathrm{g} / \mathrm{ml}$ Blasticidin S $\mathrm{HCl}$. After two weeks of growth in selective media, the clone with green fluorescence was selected and continually cultured in the normal medium. Cells were subjected to SDS-PAGE and 
A

\begin{tabular}{ccc}
\hline Cell line & $\mathrm{IC}_{50}(\mathrm{~mol} / \mathrm{I})$ & $\mathrm{RI}$ \\
\hline MX-1 & $2.02 \times 10^{-8}$ & \\
MX-1/Taxol & $2.89 \times 10^{-6}$ & 142.97 \\
MCF-7 & $1.23 \times 10^{-10}$ & \\
MCF-7/Taxol & $2.32 \times 10^{-8}$ & 268.13 \\
\hline
\end{tabular}

C

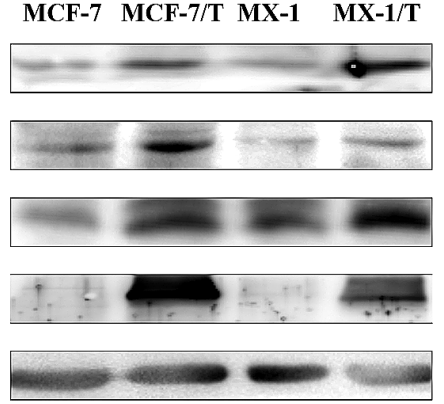

Aurora A

p-Aurora A

p-Histone $\mathrm{H3}$

P-gp

Actin
B

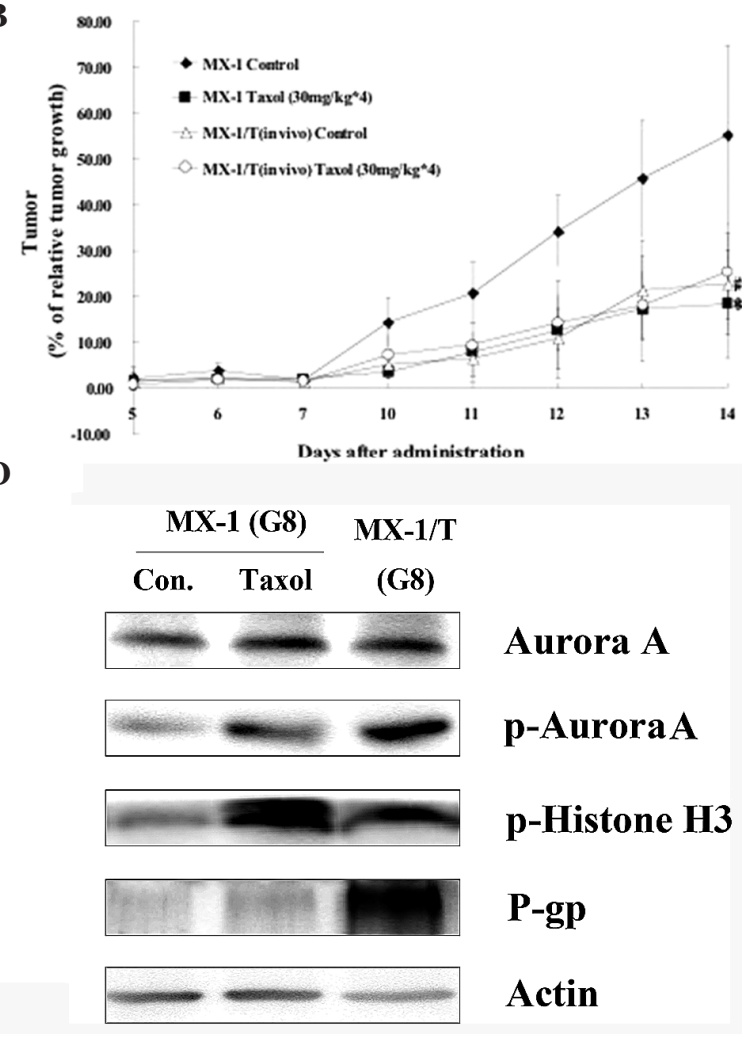

Figure 1. Expression and action of Aurora kinase A in Taxol-resistant breast cancer in vitro and in vivo. (A) After treatment with different concentrations of Taxol for $96 \mathrm{~h}$, cell viability was determined using MTT assay. The viability of the untreated cells was regarded as $100 \%$. From this assay, MCF-7/Taxol and MX-1/Taxol cells appear to be more resistant to Taxol. (B) Establishment of Taxol-resistant MX-1 xenograft model. Tumor resistance was achieved in the flanks of Athymic nude mice after eight passages in vivo. The nude mice were treated with $30 \mathrm{mg} / \mathrm{kg} / \mathrm{day} \mathrm{x} 1 \mathrm{Taxol}$ on each 14 -day passage. Resistance was characterized by the absence of tumor regression and a significant decrease of tumor-doubling time. "P<0.05, the group of Taxol treatment compared to the control group in the MX-1 xenograft model; ${ }^{\#} \mathrm{P}<0.05$, the resistant MX-1 group compared to the parent MX-1 group. (C) Western blot analysis of Aurora expression in MX-1/Taxol, MX-1, MCF-7/Taxol and MCF-7 cells. Cells were harvested to prepare cell lysates. The lysates were subjected to SDS-PAGE and blotted with antibody. The expression of p-Aurora A and p-Histone H3 was higher in MCF-7/Taxol and MX-1/Taxol cells. (D) Western blot analysis of Aurora expression from xenografted tumors. Extracted tumor proteins ( $80 \mu \mathrm{g} /$ lane, mixed from 6 samples per group) were subjected to SDS-PAGE and blotted with antibody. A high expression of p-Aurora A and p-Histone H3 was observed in the Taxol-resistant xenograft group and Taxol-treated parent MX-1 xenograft group.

harvested for Western blot analysis was performed to determine Aurora kinase A and phosphorylated activation.

Sensitivity of knockdown cells in the xenograft model. Athymic nude mice (Balb/c-nu/nu females, 6-8 weeks old) were used in the experiment. Each cell line was trypsinized and washed twice with PBS. Cells $\left(3 \times 10^{6}\right)$ suspended in $200 \mu \mathrm{l}$ $0.9 \% \mathrm{NaCl}$ were injected subcutaneously into the left flank of Balb/c-nu/nu mice. Five days after injection, 6 mice were maintained without treatment, whereas 6 others were injected with $30 \mathrm{mg} / \mathrm{kg}$ Taxol intraperitoneally three times within 1 week. Tumor growth was measured daily and the volume calculated as length $\mathrm{x}$ width $\mathrm{x}$ depth $\mathrm{x} 0.5$.

Statistical analysis. Data are expressed as the means \pm standard deviation. Statistical analysis of the results was performed using the Student's t-test. $\mathrm{P}<0.05$ was considered to be statistically significant.

\section{Results}

Status and activation of Aurora kinase A in Taxol-resistant breast cancer in vitro and in vivo. This study was conducted with the hypothesis that Aurora kinase A status and activation was correlated with resistance in breast cancer to Taxol through P-gp.

First, MTT assay was performed to determine the chemoresistance of MX-1/Taxol and MCF-7/Taxol cells to Taxol. The human breast cancer cell MX-1/Taxol is a Taxol-resistant cell line derived from the Taxol-sensitive parent cell line, MX-1. The MCF-7/Taxol cell line was established from its parent cell line MCF-7 in the lab (described in Materials and methods). MX-1/Taxol and MCF-7/Taxol cells exhibited a significant increase in $50 \%$ inhibitory concentration $\left(\mathrm{IC}_{50}\right)$ to Taxol, as analyzed by MTT assay. The $\mathrm{IC}_{50}$ values for MX-1/ Taxol and MCF-7/Taxol cells were $2.89 \mu \mathrm{mol} / 1$ and $23.2 \mathrm{nM}$. These were 142.97- and 268.13-fold higher than those of the parental cells $(20.2 \mathrm{nmol} / \mathrm{l}$ for MX-1 cells and $0.123 \mathrm{nmol} / 1$ for MCF-7 cells), as shown in Fig. 1A.

To assess drug resistance for the Taxol in vivo model and study resistance mechanisms correlated with Aurora kinase A obtained in a therapeutic setting, a xenograft model of human Taxol-resistant MX-1 tumor was developed. Tumor resistance was achieved after eight passages in vivo in nude mice treated with $30 \mathrm{mg} / \mathrm{kg}$ Taxol prior to sacrifice $(24 \mathrm{~h})$; each passage was measured every 14 days. Resistance was characterized by the 
A

MCF-7 MCF-7/T MX-1 MX-1/T

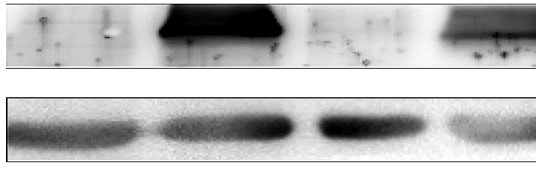

P-gp

Actin

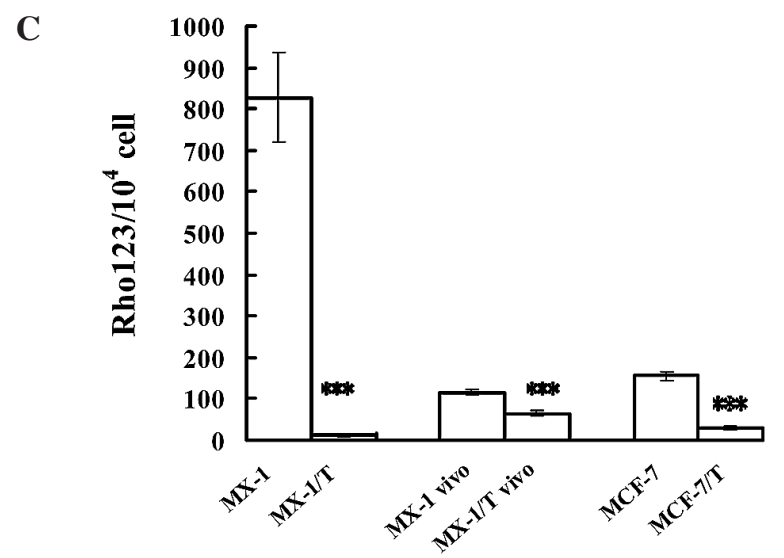

B

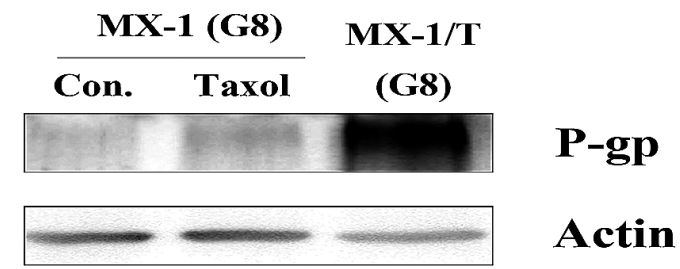

D

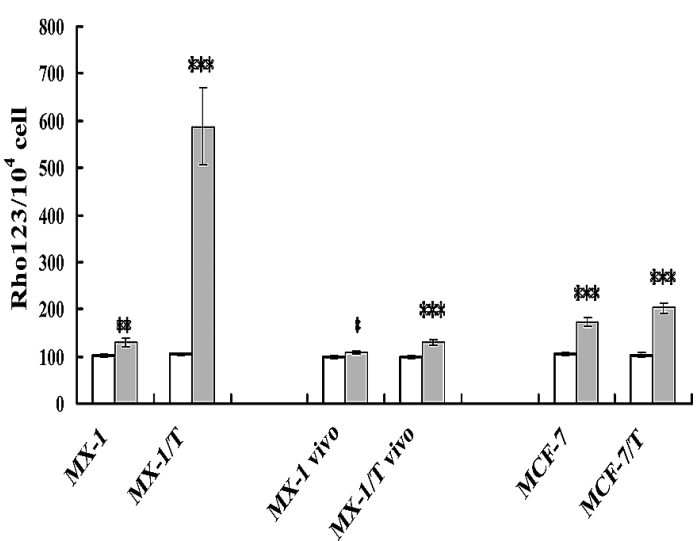

Figure 2. Increased expression and action of P-gp in a Taxol-resistant model in vitro and in vivo. (A) Western blot analysis of P-gp expression levels in whole-cell lysates in breast-resistant cancer cells and their parent cancer cells. The expression of P-gp was significantly high in resistant cells. (B) Western blot analysis of P-gp expression levels in MX-1 and MX-1/Taxol xenograft tumor tissues. The expression of P-gp was found to be high in resistant xenograft tumors. The P-gp expression was increased in Taxol-treated parent MX-1 xenograft tumors. (C) FCM analysis of the Rho123 accumulation in cells. Following incubation of breast cancer cells with $10 \mu \mathrm{mol} / 1 \mathrm{Rho} 23$ fluorescence for $3 \mathrm{~h}$, the cell Rhol23 fluorescence values were measured using the excitation and emission wavelengths of 480 and $540 \mathrm{~nm}$, respectively; 10,000 cells were calculated per sample. Data are the means \pm SD of three separate samples (significance vs. the parent cells, ${ }^{*} \mathrm{P}<0.05 ;{ }^{* * *} \mathrm{P}<0.01,{ }^{* * * *} \mathrm{P}<0.001$ ). The Rho1 23 fluorescence was much lower in resistant cells. (D) Flow cytometry of the Rhol23 accumulation in cells treated with Verapamil. Following incubation of breast cancer cells with $10 \mu \mathrm{mol} / 1$ Rhol 23 and $10 \mu \mathrm{mol} / \mathrm{l}$ Verapamil for $3 \mathrm{~h}$, the cell Rho123 fluorescence values were measured using the excitation and emission wavelengths of 480 and $540 \mathrm{~nm}$, respectively; 10,000 cells were calculated per sample. Data are the means $\pm \mathrm{SD}$ of three separate samples (significance vs. the parent cells, ${ }^{*} \mathrm{P}<0.05 ;{ }^{* * *} \mathrm{P}<0.01,{ }^{* * * *} \mathrm{P}<0.001$ ). Verapamil may reverse the activity of P-gp in Taxol-resistant breast cancer.

absence of tumor regression. In comparison to the parental Taxol-sensitive MX-1 tumors in the xenograft model, Taxol $(30 \mathrm{mg} / \mathrm{kg}$, three times each week) failed to induce growth inhibition in the MX-1/Taxol tumors. As shown in Fig. 1, the resistance test of Generation 8 to Taxol, the RTV of MX-1 was $55.31 \pm 19.57$ (control) vs. $18.39 \pm 11.90$ (Taxol treatment). However, the RTV of MX-1/Taxol was 22.72 \pm 11.04 (control) vs. $25.41 \pm 10.40$ (Taxol treatment). From these data, MX-1/Taxol was observed to grow more slowly than the MX-1 tumors.

The above data indicate that MX-1/Taxol and MCF-7/ Taxol cells, as well as the MX-1-resistant xenograft model, gained significant resistance to Taxol in vitro and in vivo, respectively. Both are suitable for application in further study.

Numerous studies have reported that the expression- and phosphorylation-mediated activation of Aurora kinase A plays a crucial role in a variety of cancers, such as prostate, colorectal, ovarian and breast cancer $(9,10)$. Therefore, the status and activity of Aurora kinase A in resistant breast cancer would be of great interest. To test whether Aurora kinase A expression was involved in Taxol resistance, the Aurora A expression levels and phosphorylation were analyzed using Taxol-resistant MX-1 and MCF-7 cells.

The activation level of Aurora kinase A was estimated via the level of p-Aurora A and p-Histone H3 (11). Western blotting (Fig. 1C) results revealed that the levels of protein expression and phosphorylation were higher in MX-1/Taxol and MCF-7/Taxol cells than in the parent MX-1 and MCF-7 cells. Differences in the activation of Aurora kinase A may thus be correlated with resistance in MX-1- and MCF-7resistant cells.

To confirm this hypothesis, the status and activation of Aurora kinase A in tumors resected from mice (the 8th passage xenograft with or without treatment with Taxol) were measured. Fig. 1D shows that, in sensitive xenograft tumor tissue, the expression of p-Aurora A and p-Histone $\mathrm{H} 3$ was higher in the Taxol-treated group than in the control group. This discrepancy reveals that Taxol may increase the activation of Aurora A. Compared to sensitive tumors, the resistant tumors showed a markedly high level of Aurora A, and phosphorylation of Aurora kinase A and Histone $\mathrm{H}$ 3, suggesting a higher activation of Aurora kinase A in the resistant tumors. Aurora kinase A likely played a crucial role in this Taxol-resistant model.

Increased expression and action of P-gp in MX-1/Taxol and $M C F-7 / T a x o l$ model in vitro and in vivo. The expression of P-gp is known to be encoded by the MDR1 gene (12). A number of studies have supported P-gp expression in tumor cells correlating with poor prognosis of chemotherapy (13). The multidrug resistance transporter (MDR) is associated with resistance to Taxol (14); one such MDR is P-gp. Taxol is known as a P-gp substrate, and cells are resistant to Taxol after overexpression to P-gp. Therefore, Western blot analysis 


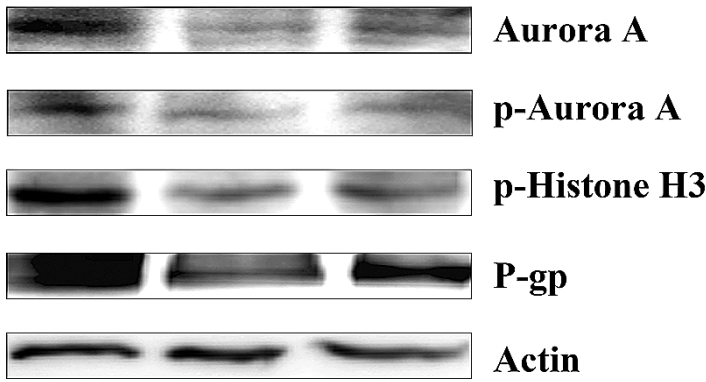

B

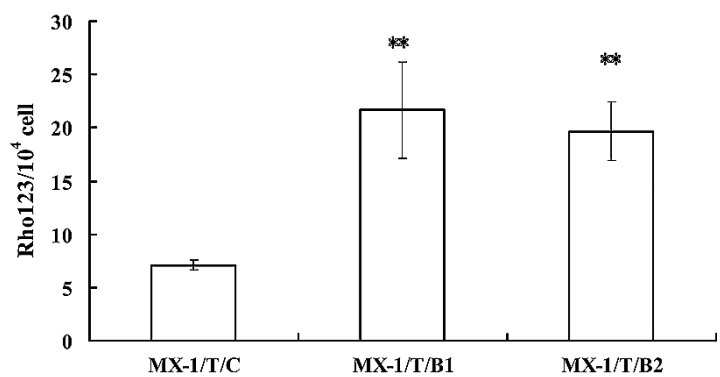

Figure 3. Silencing of Aurora kinase A inhibited the expression and action of P-gp in MX-1/Taxol cells. (A) Western blot analysis of P-gp expression levels in whole-cell lysates in MX-1/Taxol silence of Aurora A cells. MX-1/T/C are the MX-1/Taxol cells transfected with control miRNA. MX-1/T/A1 and MX-1/T/A2 are the MX-1/Taxol cells transfected with miRNA-targeted Aurora A. The expression of P-gp in MX-1/T/A1 and MX-1/T/A2 cells was found to be reduced (B) Following incubation of MX-1/T/C, MX-1/T/A1 and MX-1/T/A2 cells with $10 \mu$ mol/1 Rho123 for 3 h, the Rho123 fluorescence values in cells were measured as before. Rho123 fluorescence was higher in MX-1 cells transfected with miRNA-targeted Aurora $\mathrm{A}\left({ }^{*} \mathrm{P}<0.05 ;{ }^{* *} \mathrm{P}<0.01\right.$ and $\left.{ }^{* * * *} \mathrm{P}<0.001\right)$.

was used to determine P-gp expression in MX-1, MX-1/Taxol, MCF-7 and MCF-7/Taxol cells. As shown in Fig. 2A, P-gp protein was highly expressed in MX-1/Taxol and MCF-7/ Taxol cells. On the other hand, the P-gp protein did not exhibit as obvious an expression in wild-type MX-1 and MCF-7 cells.

The results in the xenograft-resistant model are similar to those in vitro. The expression of P-gp increased slightly after the administration of Taxol, since Taxol is thought to be the substrate of P-gp. The tumors from the xenograft-resistant mice expressed a high level of P-gp (Fig. 2B).

The transport activity of P-gp in the Rho123 accumulation assay was further examined. In MX-1/Taxol and MCF-7/Taxol cells, the intracellular accumulation of Rho123, a substrate of MDR1, decreased in comparison to parental MX-1 and MCF-7 cells (Fig. 2C). However, in the presence of $10 \mu \mathrm{M}$ Verapamil (a P-gp inhibitor), the Rho123 accumulation deficits in MX-1/ Taxol and MCF-7/Taxol were partly reversed (Fig. 2D).

The involvement of P-gp in the xenograft tumor was also determined. The tissue culture of tumors from the xenograft model was processed, after which the activity of P-gp was determined by the Rho123 accumulation assay with FCM. As shown in Fig. 2C and D, the accumulation of Rho123 was lower in the Taxol-resistant MX-1 xenograft model than in the parent MX-1 model. Verapamil is able to reverse this trend slightly.

P-gp was confirmed to have a high expression and functionality in these Taxol-resistant cells and tumors.

Generation of stable knockdown Aurora kinase A in MX-1/ Taxol cells. According to the above data, the status and activation of the Aurora kinase A in resistant cells were determined to increase. Aurora kinase A was shown to play a significant role in resistance in breast cancer cells. In order to further clarify the relationship between the activation of Aurora kinase A and Taxol resistance in breast cancer cells, MX-1/Taxol cells were transfected with a vector containing human-specific Aurora A miRNA or a control miRNA. To determine whether RNAi induction may be specifically useful for the inhibition of Aurora A, four miRNAs were generated, each targeting specific regions of the Aurora A. A number of independent clones, in which the vector containing Aurora A was introduced, were established. Two of these clones
(MX-1/Taxol/A1 and MX-1/Taxol/A2) were selected from the different miRNA-targeting Aurora A for further studies. Results of Western blot analysis revealed that Aurora A decreased significantly in cells stably expressing the Aurora A miRNA compared to cells expressing control miRNA (MX-1/ Taxol/C). Aside from the expression of Aurora kinase A, phosphorylated activation of Aurora kinase A and Histone H3 decreased significantly after Aurora kinase A knockdown. This indicates that knockdown MX-1/Taxol cells targeting Aurora A were successfully generated (Fig. 3A).

Silencing of Aurora kinase A inhibition of the expression and function of $P$-gp in MX-1/Taxol cells. Using the MX-1/Taxol cells stably transfected with Aurora A miRNA, the effect of Aurora kinase A inhibition on P-gp expression and function was assessed. Western blotting (Fig. 3A) revealed that the level of P-gp significantly decreased in Aurora kinase A knockdown cells (MX-1/Taxol/A1 and MX-1/Taxol/A2) as compared to the control cell line MX-1/Taxol/C.

To observe the effects of Aurora kinase A inhibition on P-gp function, Rho123 accumulation was again selected. Results of the flow cytometry (Fig. 3B) showed that the intercellular accumulation of Rho123 in the MX-1/Taxol cells silencing Aurora A was higher than that of MX-1/Taxol cells transfected with control miRNA. This elevation reveals that knockdown of Aurora A not only markedly decreased the expression of P-gp, but also down-regulated the P-gp function in resistant breast cancer cells.

Aurora kinase A was shown to partly mediate Taxol resistance through the modulation of P-gp.

p-ERK-mediated down-regulation of P-gp in MX-1/Taxol cells through Aurora A inactivation. Fig. 4A shows that Western blot analysis revealed that the phosphorylation of ERK2 was higher in MX-1/Taxol and MCF-7/Taxol cells than in the parent cells. However, no significant difference was found in the expression of ERK2.

In the xenograft model (Fig. 4B), the treatment of Taxol resulted in a high expression of p-ERK2. On the other hand, Taxol did not affect the expression ERK2 in mice-bearing MX-1 tumors. Simultaneously, the expression of p-ERK2 was significantly higher in the Taxol-resistant tumors than that in 
A
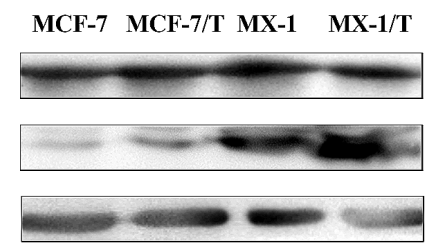

B

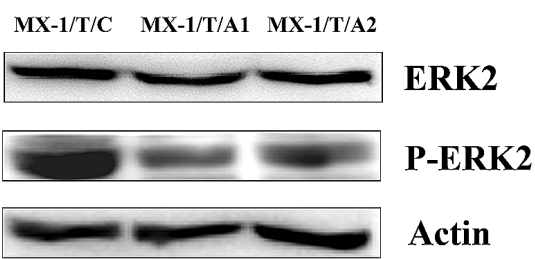

C MX-1 (G8) MX-1/T

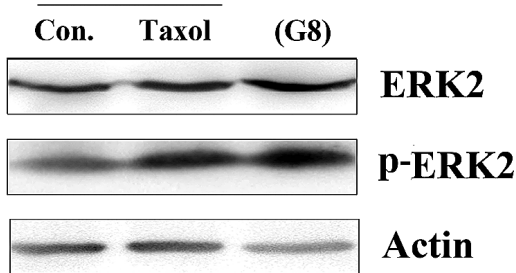

Figure 4. p-ERK-mediated down-regulation of P-gp in MX-1/Taxol cells through Aurora A inactivation. (A) Western blot analysis of p-ERK2 and ERK2 expression levels in whole cell lysates in resistant breast cancer cells and their parent cancer cells. The phosphorylation of ERK2 was higher in resistant cells. (B) Western blot analysis of p-ERK2 and ERK2 expression levels in whole cell lysates from the xenograft tumor tissues. Whole tissue lysates were obtained using lysis buffer. The lysates were subjected to Western blotting with the indicated antibodies. The expression of p-ERK2 was higher in resistant xenograft tumors. (C) Western blot analysis of p-ERK2 and ERK2 expression levels in whole-cell lysates in MX-1/Taxol silence of Aurora A cells. MX-1/T/C are the MX-1/Taxol cells transfected with control miRNA. MX-1/T/A1 and MX-1/T/A2 are the MX-1/Taxol cells transfected with miRNA-targeted Aurora A. The down-regulation of p-ERK2 was in silencing Aurora A cells.
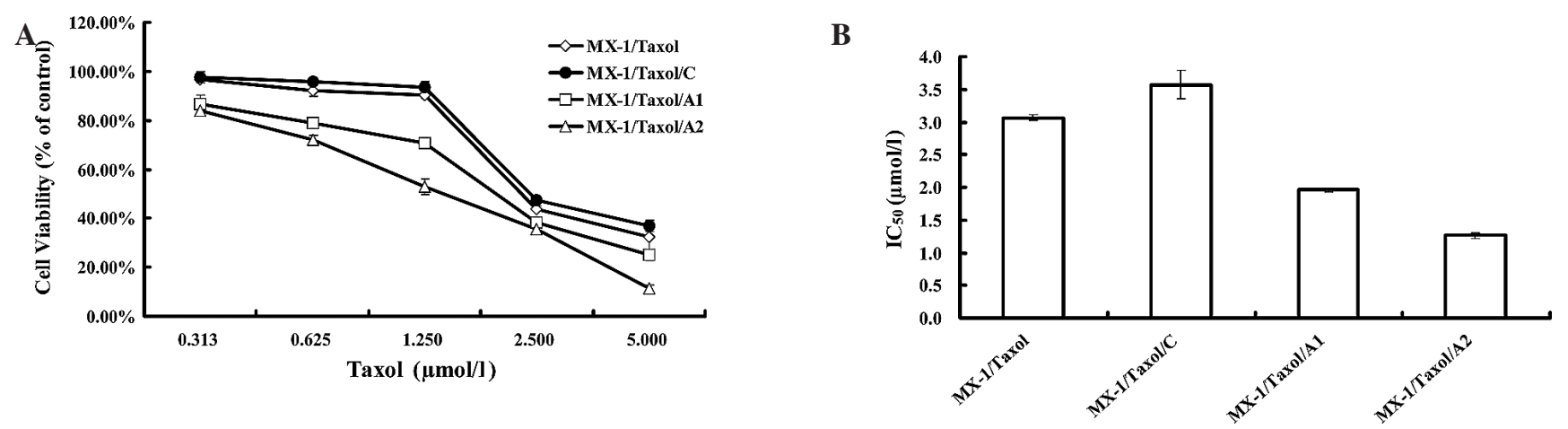

Figure 5. Silencing of Aurora kinase A partially rescued Taxol sensitivity in vitro. MX-1/T/C, MX-1/T/A1 and MX-1/T/A2 cell treatment with different concentrations of Taxol for $96 \mathrm{~h}$ is shown. Cell viability was determined using the MTT assay. The viability of the untreated cells was regarded as $100 \%$. Points are the mean of three independent experiments; bars are SD. (A) The proliferation curve of MX-1/T/C, MX-1/T/A1 and MX-1/T/A2 cells treated with different concentrations of Taxol for $96 \mathrm{~h}$ is shown. (B) The $\mathrm{IC}_{50}$ vlaues of MX-1/T/C, MX-1/T/A1 and MX-1/T/A2 cells treated with different concentrations of Taxol for $96 \mathrm{~h}$ are shown. MX-1/T/A1 and MX-1/T/A2 cells were found to be more sensitive to Taxol than MX-1/T/C cells.

the sensitivity tumors, whereas no change was noted in the expression of ERK, similar to the in vitro results.

The role of the ERK pathway in the generation of multidrug resistance has received more consideration than in the past. Previous studies demonstrated that the modulation of ERK activation may be a novel method used to reverse multidrug resistance (15). In the present study, the changing pattern of p-ERK was similar to that of $\mathrm{p}$-Aurora A and P-gp; accordingly, $\mathrm{p}$-Aurora $\mathrm{A}$ is proposed to regulate $\mathrm{P}$-gp through p-ERK. To test this hypothesis, the expression and activation of ERK2 in the silencing Aurora A MX-1/Taxol sublines were observed. Western blotting results revealed that the phosphorylation of ERK2 was lower in MX-1/Taxol/A1 and MX-1/Taxol/A2 than in the cells transfected with control miRNA. However, no change was found in the expression of ERK2 (Fig. 4C). This lack of change reveals that silencing Aurora A may decrease the phosphorylation of ERK2, and modulate P-gp. However, Aurora A did not affect the expression of ERK2.

Silencing of Aurora kinases in partial rescue of Taxol sensitivity in vitro and in vivo. Since the relationship of Aurora A status and activation with resistance to Taxol was established, inhibition of Aurora kinase A was thought to result in enhanced sensitivity of MX-1/Taxol to Taxol. To prove this hypothesis, MX-1/Taxol cells transfected with miRNA were treated with various doses of Taxol. As expected, MX-1/Taxol/ A1 and A2 were more sensitive to Taxol than MX-1/Taxol and MX-1/Taxol/C (i.e., $\mathrm{IC}_{50}$ values of Taxol in MX-1/Taxol/A1 and A2 were reduced by 45.1 and $64.5 \%$, respectively, compared to those in MX-1/Taxol and MX-1/Taxol/C) (Fig. 5).

As described above, the outcomes of the in vitro experiments with the silencing of Aurora kinase A were nearly identical. Studies were then performed in vivo to determine the differences in growth and sensitivity to Taxol among the MX-1/ Taxol sublines (MX-1/Taxol/C, MX-1/Taxol/A1 and MX-1/ Taxol/A2). Each cell line was injected subcutaneously into nude mice. The growth of MX-1/Taxol/A1 and MX-1/Taxol/A2 tumors was found to be significantly slower than that of MX-1/ Taxol/C. The differences in Taxol-induced growth inhibitory effects among MX-1/Taxol sublines were then compared in vivo. In the preliminary study, the growth of MX-1/Taxol tumors was not inhibited by treatment with $30 \mathrm{mg} / \mathrm{kg}$ Taxol three times a week. Fig. 6B shows that growth of MX-1/ Taxol/C after treatment with Taxol was similar to that without Taxol treatment, whereas MX-1/Taxol/A1 and MX-1/Taxol/ A2 tumors grew slowly after Taxol treatment (Fig. 6C and D) compared to tumors without the administration of Taxol. The 
A

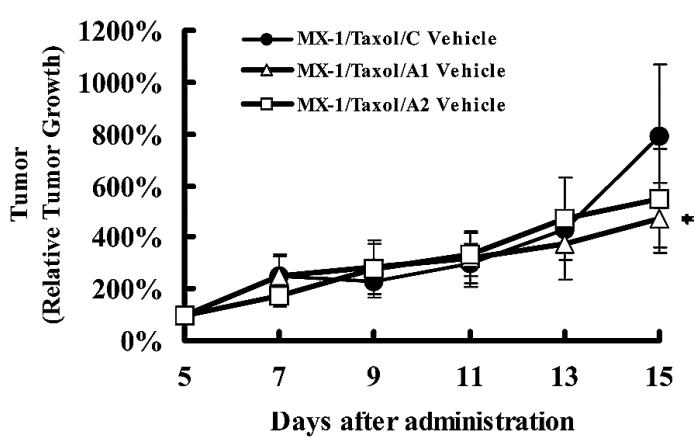

C

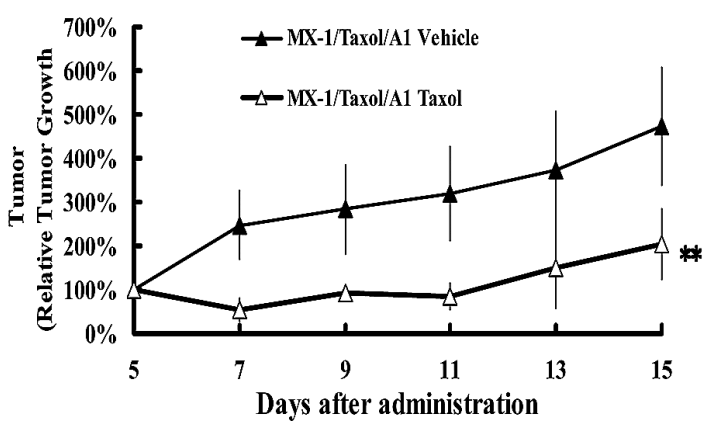

B

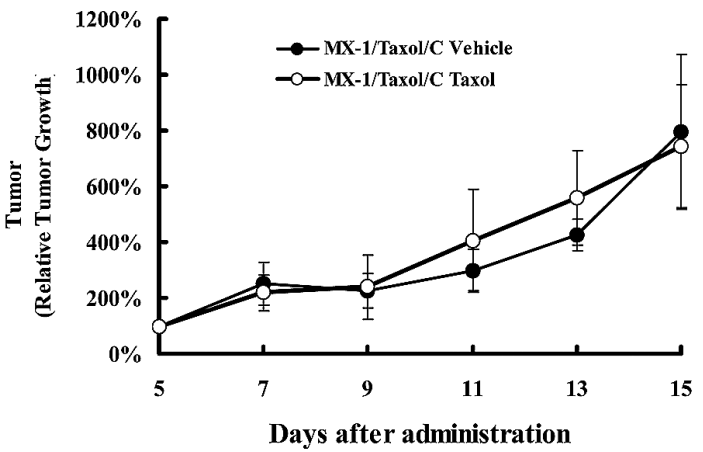

D

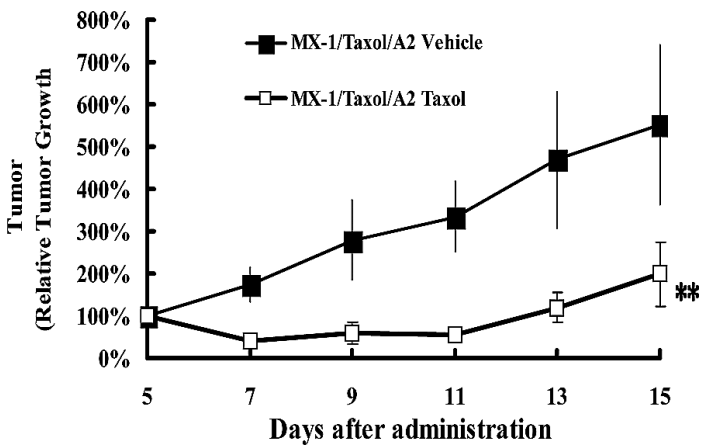

Figure 6. Silencing of Aurora kinase A partially rescued Taxol sensitivity in vivo. (A) Changes in the volume of MX-1/Taxol/C, MX-1/Taxol/A1 and MX-1/ Taxol/A2 xenograft tumors in mice. Cells were injected subcutaneously into female nude mice. The tumor dimension was measured daily after 5 days of injection. Each point is the mean (bar, SD) of independent measurements of tumor size. Percentage of relative tumor growth is calculated as the mean tumor volume for each day divided by the volume at the time of the first measurement. The growth of MX-1/T/A1 and MX-1/T/A2 was much slower compared to MX-1/T/C xenograft tumors ( $\left.\mathrm{P}<0.05 ;{ }^{* *} \mathrm{P}<0.01,{ }^{* * *} \mathrm{P}<0.001\right)$. (B-D) Changes in the volume of (B) MX-1/Taxol/C, (C) MX-1/Taxol/A1 and (D) MX-1/Taxol/A2 xenograft tumors in mice after Taxol administration. Cells were injected subcutaneously into female nude mice; 5 days later, Taxol was administered three times in 1 week by intraperitoneal injection. The tumor dimension was measured daily after the first dose. Each point is the mean (bar, SD) of independent measurements of tumor size. The percentage of relative tumor growth is calculated as the mean tumor volume on each day divided by the volume at the time of the first Taxol or vehicle administration ( ${ }^{*} \mathrm{P}<0.05 ;{ }^{* *} \mathrm{P}<0.01$ and $\left.{ }^{*}{ }^{* *} \mathrm{P}<0.001\right)$. MX-1/Taxol/C xenograft tumors did not respond to Taxol. However, MX-1/ Taxol/A1 and MX-1/Taxol/A2 xenograft tumors were found to be sensitive to Taxol.

inhibition rate of Taxol in MX-1/Taxol/A1 and MX-1/Taxol/ A2 was 56.81 and $63.77 \%$, respectively, (according to the RTV) 14 days after tumor cell injection, indicating that MX-1/ Taxol/A1 and MX-1/Taxol/A2 were less resistant to Taxol than $\mathrm{MX}-1 /$ Taxol/C in vivo.

Collectively, the above analysis reveals that resistance to Taxol decreased by the inhibition of Aurora kinase A in breast cancer.

\section{Discussion}

MDR-associated multidrug resistance has often been associated with the intrinsic or acquired chemoresistance to antitumor agents. Since Taxol is a substrate of P-gp, increased activity of drug efflux via overexpressed P-gp has been proposed as one of the mechanisms of Taxol resistance (16). Therefore, extensive research has been conducted to identify MDR1 inhibitors to reverse chemoresistance to Taxol. However, severe side effects have been noted in the modulators of MDR1 transport activity that affect the kinetics of other drugs (17).

Considering that the down-regulation of MDR1 gene expression may be an alternative way of enhancing the sensitivity of chemotherapy, elucidating the pathway-regulated MDR1 is crucial in the identification of potential therapeutic targets to overcome chemoresistance. Therefore, in addition to inves- tigating the role of Aurora kinase $\mathrm{A}$ in the regulation of P-gp expression in resistant breast cancer, the present study evaluated the effect of Aurora kinase A in overcoming chemoresistance.

In this study, the activation of Aurora kinase A was higher in resistant breast cancer cells. This was proven in vitro and in vivo simultaneously. The up-regulation of Aurora A activation was observed with increasing Taxol resistance, suggesting that Aurora kinase A may yield the phenotype of drug resistance. Taxol is known to increase the phosphorylation of Aurora kinases. Thus, the high activation of Aurora A may be caused by the effect of long-term administration of Taxol in resistant cells and nude mice bearing MX-1. However, the detailed mechanism remains to be determined. Various studies $(18,19)$ have recently reported similar findings on the correlation of Aurora kinases to chemosensitivity. However, to the best of our knowledge, the high expression of Aurora A has rarely been reported in resistant breast xenograft model.

The in vitro studies indicated that the overexpression and action of $\mathrm{P}-\mathrm{gp}$ is associated with resistance to Taxol in MCF-7/ Taxol and MX-1/Taxol cells. The specific miRNA targeting Aurora kinase A was introduced to elucidate the potential role of Aurora kinase A in P-gp induction in the Taxol-resistant MX-1 cells. P-gp expression and function was potently decreased after silencing Aurora kinase A, emphasizing the significance of Aurora kinase A for P-gp regulation. Studies 
investigating the correlation between the phosphorylation of Aurora A and chemoresistance to Taxol through P-gp remain rare; thus, further investigations are imperative.

P-gp expression in the MDR1-transduced MCF-7/MDR and MDA-MB-231/MDR human breast cancer cell lines has recently been found to be positively regulated by the ERK pathway. Inhibition of the MDK-ERK-RSK pathway decreases cell surface P-gp expression by promoting degradation. In addition, the modulation of ERK activation has been suggested to reverse multidrug resistance in prostate, gastric and hematopoietic cancer $(20,21)$. Similarly, in the present study, the phosphorylation of ERK2 was higher in MCF-7/Taxol, MX-1/ Taxol cells, as well as in the Taxol-resistant xenograft MX-1, and was down-regulated by Aurora A silencing. Therefore, Aurora A may regulate P-gp through p-ERK2. However, the relationship of p-ERK2 with the expression and function of P-gp in Taxolresistant breast cancer requires detailed future investigation.

Aurora kinase function shows complex regulation, depending on cell/tissue type, differentiation status and environment. Previous studies have shown that a high expression of Aurora A interferes with the Mad2-Cdc20 signal and overrides the mitotic checkpoint, even in the presence of defective spindle formation. This process suggests that a high-level expression of Aurora kinases A attenuates the antitumor activity of docetaxel $(22,23)$, indicating that Aurora kinase A affects resistance to Taxol through alternative pathways.

Findings of the present study showed the effect of Aurora A on the sensitivity to Taxol in resistant breast cancer in vitro and in vivo. Results of the transfection experiments targeting Aurora kinase A indicate that Taxol-resistant MX-1 cells became more sensitive to Taxol after silencing Aurora kinase A. Consistent with the in vitro studies, results of in vivo experiments with the MX-1/Taxol tumors with silenced Aurora kinase A exhibited significant growth suppression and enhanced sensitivity to Taxol compared to those in control MX-1/Taxol that lacked growth inhibition. Although knockdown of Aurora kinase A by miRNA may partly rescue Taxol sensitivity, it was insufficient to completely restore Taxol sensitivity, which may be due to the fact that alterations in genes other than Aurora A confer the chemosensitivity in resistant breast cancer to Taxol, such as BRCA1, tau and tubulin (24).

Taken together, Aurora kinase A is associated with tumor resistance and plays a crucial role in Taxol resistance breast cancer partly through $\mathrm{p}$-ERK/P-gp. Therefore, inhibition of Aurora kinase A may facilitate Taxol therapy in resistant breast cancer.

The present study focuses mainly on the resistant threetriple negative breast cancer. However, more and detailed research should be conducted on the role of Aurora kinase A in ER-positive resistant breast cancer.

Although Aurora kinase A should be explored and investigated with regards to the Taxol-resistant mechanisms to Taxol, Aurora kinase A serves as a potential therapeutic target for overcoming chemoresistance and increasing sensitivity in breast cancer to Taxol.

\section{References}

1. Moulder S and Hortobagyi GN: Advances in the treatment of breast cancer. Clin Pharmacol Ther 83: 26-36, 2008.
2. Newman SP, Foster PA, Stengel C, et al: STX140 is efficacious in vitro and in vivo in taxane-resistant breast carcinoma cells. Clin Cancer Res 14: 597-606, 2008.

3. Patel N, Chatterjee SK, Vrbanac V, et al: Rescue of paclitaxel sensitivity by repression of Prohibitin 1 in drug-resistant cancer cells. Proc Natl Acad Sci USA 107: 2503-2508, 2010.

4. Zhou J, Liu M, Aneja R, Chandra R, Lage H and Joshi HC: Reversal of P-glycoprotein-mediated multidrug resistance in cancer cells by the c-Jun NH2-terminal kinase. Cancer Res 66: 445-452, 2006

5. Payton M, Bush TL, Chung G, et al: Preclinical evaluation of AMG 900, a novel potent and highly selective pan-aurora kinase inhibitor with activity in taxane-resistant tumor cell lines. Cancer Res 70: 9846-9854, 2010.

6. Lapenna S and Giordano A: Cell cycle kinases as therapeutic targets for cancer. Nat Rev Drug Discov 8: 547-566, 2009.

7. Kumano M, Miyake H, Terakawa T, Furukawa J and Fujisawa M: Suppressed tumour growth and enhanced chemosensitivity by RNA interference targeting Aurora-A in the $\mathrm{PC} 3$ human prostate cancer model. BJU Int 106: 121-127, 2010.

8. Li Y, Zhang ZF, Chen J, et al: VX680/MK-0457, a potent and selective Aurora kinase inhibitor, targets both tumor and endothelial cells in clear cell renal cell carcinoma. Am J Transl Res 2: 296-308, 2010.

9. Gautschi O, Heighway J, Mack PC, Purnell PR, Lara PN Jr and Gandara DR: Aurora kinases as anticancer drug targets. Clin Cancer Res 14: 1639-1648, 2008.

10. Katayama H, Brinkley WR and Sen S: The Aurora kinases: role in cell transformation and tumorigenesis. Cancer Metastasis Rev 22: 451-464, 2003.

11. Crosio C, Fimia GM, Loury R, et al: Mitotic phosphorylation of histone H3: spatio-temporal regulation by mammalian Aurora kinases. Mol Cell Biol 22: 874-885, 2002.

12. Nobili S, Landini I, Giglioni B and Mini E: Pharmacological strategies for overcoming multidrug resistance. Curr Drug Targets 7: 861-879, 2006.

13. Guo X, Ma N, Wang J, et al: Increased p38-MAPK is responsible for chemotherapy resistance in human gastric cancer cells. BMC Cancer 8: 375, 2008

14. Szakacs G, Paterson JK, Ludwig JA, Booth-Genthe C and Gottesman MM: Targeting multidrug resistance in cancer. Nat Rev Drug Discov 5: 219-234, 2006.

15. Katayama K, Yoshioka S, Tsukahara S, Mitsuhashi J and Sugimoto Y: Inhibition of the mitogen-activated protein kinase pathway results in the down-regulation of P-glycoprotein. Mol Cancer Ther 6: 2092-2102, 2007.

16. Litman T, Druley TE, Stein WD and Bates SE: From MDR to MXR: new understanding of multidrug resistance systems, their properties and clinical significance. Cell Mol Life Sci 58: 931-959, 2001.

17. Han CY, Cho KB, Choi HS, Han HK and Kang KW: Role of FoxO1 activation in MDR1 expression in adriamycin-resistant breast cancer cells. Carcinogenesis 29: 1837-1844, 2008.

18. Hua Yang, Lili He, Patricia Kruk, et al: Aurora-A induces cell survival and chemoresistance by activation of Akt through a p53-dependent manner in ovarian cancer cells. Int J Cancer 119: 2304-2312, 2006.

19. Ayman El-Sheikh, Rong Fan, Diane Birks, et al: Inhibition of Aurora kinase A enhances chemosensitivity of medulloblastoma cell lines. Pediatr Bloof Cancer 55: 35-41, 2010.

20. Li Y, Li S, Han Y, et al: Calebin-A induces apoptosis and modulates MAPK family activity in drug resistant human gastric cancer cells. Eur J Pharmacol 591: 252-258, 2008.

21. McCubrey JA, Steelman LS, Abrams SL, et al: Roles of the RAF/MEK/ERK and PI3K/PTEN/AKT pathways in malignant transformation and drug resistance. Adv Enzyme Regul 46: 249-279, 2006.

22. Anand S, Penrhyn-Lowe S and Venkitaraman AR: Aurora-A amplification overrides the mitotic spindle assembly checkpoint, inducing resistance to Taxol. Cancer Cell 3: 51-62, 2003.

23. Hata T, Furukawa T, Sunamura M, et al: RNA interference targeting aurora kinase a suppresses tumor growth and enhances the taxane chemosensitivity in human pancreatic cancer cells. Cancer Res 65: 2899-2905, 2005.

24. McGrogan BT, Gilmartin B, Carney DN and McCann A: Taxanes, microtubules and chemoresistant breast cancer. Biochim Biophys Acta 1785: 96-132, 2008. 